\title{
MedienPädagogik
}

Zeitschrift für Theorie und Praxis der Medienbildung

Themenheft Nr. 42: Optimierung in der Medienpädagogik.

Forschungsperspektiven im Anschluss an den 27. Kongress der DGfE

Herausgegeben von Patrick Bettinger, Klaus Rummler und Karsten D. Wolf

\section{Kommunikation und Zusammenarbeit zwischen Elternhaus und Schule mit digitalen Medien}

\author{
Forschungsstand und Forschungsagenda \\ Jasmin Bastian ${ }^{1}$ (D) und Doreen Prasse ${ }^{2}$ (D) \\ ${ }^{1}$ Johannes Gutenberg-Universität Mainz \\ 2 Pädagogische Hochschule Schwyz
}

\begin{abstract}
Zusammenfassung
BMBF und KMK fordern in aktuellen Strategiepapieren zur "Bildung in der digitalen Welt» eine Erweiterung der Kommunikation und Kooperation auf allen Ebenen der Schulgemeinschaft. Der Digitalisierung wird in diesem Zusammenhang das Potenzial zugeschrieben, den Informationsfluss und die Zusammenarbeit zwischen Lehrkräften und Eltern verbessern und eine stärkere Mitbestimmung am schulischen Leben fördern zu können. Der Beitrag diskutiert, inwiefern sich für diese Optimierungsunterstellung Hinweise in der Forschung finden lassen. Es zeigt sich, dass die Forschung in diesem Bereich noch am Anfang steht. Wichtige Fragen zur Rolle digitaler Medien in den Kommunikations- und Kooperationsprozessen zwischen Eltern und Schule, zur aktuellen Verbreitung und Verwendung digitaler Technologien und zu den dadurch bewirkten Veränderungen der Zusammenarbeit zwischen Elternhaus und Schule können derzeit nicht umfassend beantwortet werden. Im vorliegenden Beitrag werden deshalb zum einen wichtige Merkmale und Rahmenbedingungen einer gelingenden Zusammenarbeit herausgearbeitet und erste Forschungsergebnisse zu den Besonderheiten und Veränderungen einer digital unterstützten Kommunikation und Kooperation zusammengefasst. Zum anderen werden aktuelle Forschungsdesiderate beschrieben und eine Forschungsagenda für diesen zentralen Bereich von Schule entworfen.
\end{abstract}

Communication and Collaboration Between Home and School With Digital Media. Current State of Research and Research Agenda

\begin{abstract}
In current strategy papers on the issues and challenges of education in a digital world education policy makers call for an expansion of communication and cooperation at all levels of the school community (KMK 2016). In this context, digital technologies are seen
\end{abstract}


as having the potential to improve the flow of information and collaboration between teachers and parents, and to promote greater participation in school life. In this article, we discuss how evidence in recent research supports the postulated "optimization assumption» and identify current research desiderata in this field. However, research in this area is still in its beginning stages. Important questions about the role of digital media for communication and cooperation processes between parents and school, about the current use and dissemination of digital technologies, and about the resulting changes in home-school cooperation are not comprehensively addressed in present research. Therefore, in this article, we identify important characteristics and conditions for successful home-school cooperation and - in using this as a framework - summarize existing research results on digitally supported communication and cooperation between parents and school. We then describe current research desiderata and draft a research agenda for future research in this field.

\section{Einleitung}

Im März 2020 hat die COVID-19-Pandemie zu einer mehrmonatigen Schliessung der Schulen im deutschsprachigen Raum geführt und Kinder aller Altersstufen mussten schulische Lerninhalte von zu Hause aus erarbeiten. Eltern spielten im Rahmen dieses unfreiwilligen «Homeschoolings» eine zentrale Rolle und waren gerade in den unteren Jahrgangsstufen auf einmal intensiv am schulischen Leben beteiligt. Dies betraf nicht nur die Betreuung und Lernbegleitung der Kinder, sondern oft auch die Kommunikation mit der Schule (Bol 2020; Huber u. a. 2020). Die meisten Eltern gaben zwar an, dass sie von der Schule mit Informationen versorgt wurden, es zeigten sich jedoch Unterschiede darin, wie diese Kommunikation organisiert wurde, d. h. beispielsweise, inwieweit die Eltern mit den Lehrpersonen in direktem Kontakt standen und/oder die Kommunikation als verständlich und effektiv wahrnahmen (ebd.). In vielen Schulen wurde versucht, die Kommunikation und Kooperation mit den Familien auch durch digitale Medien zu unterstützen und zu verbessern. Dies gestaltete sich je nach Schule sehr unterschiedlich und reichte von einfacheren Formen der Versorgung mit Informationen und Materialien via E-Mail bis hin zur komplexen Nutzung unterschiedlicher medialer Möglichkeiten, beispielsweise in Form von Lernmanagementsystemen, Videokonferenzen etc. (Eickelmann und Drossel 2020). Nicht nur für viele Schülerinnen und Schüler, sondern auch für Lehrpersonen und Eltern war diese digital unterstützte Kommunikation und Kooperation vielfach ein Novum und brachte neue Fragen bezüglich einer (zukünftigen) effektiven Nutzung mit sich (Huber u. a. 2020).

Jedoch wird die Frage, ob und wie digitale Medien die Kommunikation und Kooperation zwischen Schule und Elternhaus unterstützen können, nicht erst in Zeiten coronabedingten Fernlernens diskutiert. Bereits seit den 2000er Jahren gibt es 
die Hoffnung und konkrete Bemühungen - wie beispielsweise durch den Aufbau von Schulinformationssystemen -, die Kommunikation und Kooperation durch digitale Medien zu verbessern (Breiter, Lange, und Stauke 2008; Welling, Breiter, und Schulz 2015).

Eine digital unterstützte Optimierung der Zusammenarbeit zwischen Elternhaus und Schule ist eine inzwischen auch von bildungspolitischer Seite hervorgebrachte Forderung. Diese begründet sich in der Hoffnung, dass eine solche Verbesserung der Zusammenarbeit Vorteile für die schulische Entwicklung der Schülerinnen und Schüler mit sich bringt. So wird im Strategiepapier der Kultusministerkonferenz zur «Bildung in der digitalen Welt» (KMK 2016) angenommen, dass die Digitalisierung neue «Kommunikationskulturen auf allen Ebenen innerhalb der Schulgemeinschaft» ermöglicht und dass digitale Netzwerkstrukturen, die z. B. Lehrkräfte, Schülerinnen und Schüler und Eltern einschliessen, den Informationsfluss beschleunigen und «zu einer umfassenderen Mitbestimmung und Teilhabe am schulischen Leben und an Schulentwicklungsprozessen beitragen» können (ebd., 9). Der Digitalisierung wird damit das Potenzial zugeschrieben, zu einer Optimierung der bisherigen Elternbeteiligung führen zu können. Trotz der zahlreichen neuen Möglichkeiten, die digitale Medien bieten, ist aktuell nicht klar, inwieweit diese Möglichkeiten in der Schulpraxis tatsächlich genutzt werden. Empirische Ergebnisse dazu sind nur wenige vorhanden. Es kann ausserdem auch kritisch hinterfragt werden, ob digitale Medien tatsächlich bestimmte positive Veränderungen bewirken oder eventuell nur die bereits in Schulen bestehenden mehr oder eben auch weniger positiven Kommunikations- und Kooperationskulturen verstärken (Selwyn u. a. 2011).

Im Kontext der Mediatisierung der gesamten Lebenswelt haben auch die Folgen der zunehmenden Digitalisierung vieler Bereiche des Lehrens und Lernens an Schulen an Bedeutung gewonnen (Tulodziecki, Grafe, und Herzig 2019; Schaumburg 2018). So sind in den vergangenen Jahren wesentliche Veränderungen der Nutzung digitaler Medien an Schulen zu verzeichnen (Drossel u. a. 2019). Insbesondere der Einzug von digitalen mobilen Geräten in Schulen bringt hier neue Potenziale, aber auch Herausforderungen mit sich (Döbeli Honegger 2017; Schaumburg und Prasse 2019). Zahlreiche Modellprojekte zur Einführung mobiler digitaler Geräte an Schulen (wie z. B. Tablets) wurden wissenschaftlich begleitet und dokumentieren die Konsequenzen für die Unterrichts- und Schulprozesse (Autorengruppe Paducation 2014; Bastian 2017; Bremer und Tillmann 2017; Eickelmann und Drossel 2020; Prasse, Egger und Honegger 2017; Prasse u. a. 2020). Allerdings wurde der Fokus bisher vorwiegend auf die Perspektiven von Lehrenden und Lernenden gerichtet - etwa auf deren Mediennutzung oder deren medienbezogene Einstellungen und Kompetenzen (Bastian und Aufenanger 2017). Grosse (inter-)nationale Vergleichsstudien nehmen neben den Perspektiven von Lehrenden und Lernenden zwar auch die Medienausstattung der Schulen, die Professionalisierung der Lehrpersonen oder 
Schulentwicklungsmassnahmen in den Blick (Eickelmann u. a. 2019), die Kommunikation und Kooperation mit digitalen Medien im schulischen Kontext - insbesondere unter dem Gesichtspunkt der Zusammenarbeit zwischen Elternhaus und Schule - wurde allerdings vor allem im deutschsprachigen Raum bisher nur unzureichend untersucht und diskutiert.

Es stellt sich also die Frage, welche Rolle digitale Medien in den Kommunikations- und Kooperationsprozessen zwischen Eltern und Lehrkräften spielen beziehungsweise spielen könnten. Inwieweit werden diese Prozesse durch digitale Medien unterstützt und/oder inwieweit verändern digitale Medien in der Folge die Ausgestaltung solcher Prozesse? Welche Technologien finden in diesem Kontext welche Art der Verwendung (z. B. E-Mail, Social Media, Lernmanagementsystem)? Welche schulischen und gesellschaftlichen Rahmenbedingungen spielen für eine möglicherweise «optimierte» Zusammenarbeit zwischen Elternhaus und Schule eine Rolle und welche gegebenenfalls neuen Anforderungen ergeben sich an die Beteiligten? Bezugnehmend auf diese Frage diskutiert der Beitrag als erstes, auf welchen Dimensionen der Kommunikation und Kooperation zwischen Elternhaus und Schule überhaupt Effekte digitaler Medien zu erwarten wären. Zweitens wird auf der Basis zentraler empirischer Forschungsbefunde herausgearbeitet, inwieweit sich Hinweise für die beschriebene Optimierungsannahme finden lassen und nachfolgend bestimmt, wo Forschungsdesiderate für die Klärung dieser Fragen bestehen. Zum Schluss werden eine Forschungsagenda entwickelt und methodische Empfehlungen herausgearbeitet.

\section{Bildungspolitische Rahmenbedingungen und Vorgaben zur Zusammenarbeit zwischen Elternhaus und Schule}

Wirft man zunächst einen Blick auf die Rahmenbedingungen und Vorgaben zur Zusammenarbeit von Schule und Elternhaus in Deutschland, so wird deutlich, dass der staatliche Erziehungsauftrag in der Schule, von dem Art. 7 Abs. 1 GG ausgeht, dem elterlichen Erziehungsrecht gleichgeordnet ist:

«Diese gemeinsame Erziehungsaufgabe von Eltern und Schule, welche die Bildung der einen Persönlichkeit des Kindes zum Ziel hat, läßt [sic!] sich nicht in einzelne Kompetenzen zerlegen. Sie ist in einem sinnvoll aufeinander bezogenen Zusammenwirken zu erfüllen.» (BVerfGE 34, 165ff. Urteil des ersten Senats vom 6. Dezember 1972)

Hinweise darauf, wie dieses Zusammenwirken theoretisch aussehen sollte, liefert der Beschluss der Kultusministerkonferenz (2018a) zur «Bildung und Erziehung als gemeinsame Aufgabe von Eltern und Schule». Im Beschluss verankert ist die Verpflichtung der Schule, «Eltern in allen Fragen zu beraten, die für die Entwicklung, den 
Leistungsstand und die Schullaufbahn ihres Kindes wichtig sind» (ebd., 4). Dies soll im Rahmen «regelmäßige[r] Kontaktaufnahmen und Beratungen» geschehen (ebd., 6). Umgekehrt geht für die Eltern mit diesem Recht die Aufgabe einher, «den schulischen Bildungs- und Erziehungsauftrag nach Kräften zu unterstützen und dafür zu sorgen, dass ihre Kinder an der Erfüllung des schulischen Auftrags mitarbeiten» (ebd., 4).

Wie die Zusammenarbeit und Kommunikation konkret ausgestaltet werden soll, wird von den Schulgesetzen der jeweiligen Länder geregelt (für einen Überblick KMK 2018b). Als mündliche Formate der Information und Beratung werden beispielsweise spezielle Elternsprechtage, Lehrer-Schüler-Eltern-Gespräche, besondere Informationsveranstaltungen und Einzelgespräche sowie die Möglichkeit einer Hospitation im Unterricht benannt (ebd.). Schriftliche Formate stellen etwa Logbücher und Elternbriefe dar. Einige Länder legen hier einen konkreten Mindestumfang an Beratungsangeboten fest; Hamburg beispielsweise empfiehlt mindestens zwei Elternabende pro Schuljahr (ebd.). Die konkreten Verantwortlichkeiten und Zuständigkeiten von Eltern und Schule sind jedoch nicht eindeutig definiert und unterliegen der Aushandlung und individuellen Ausgestaltung der schulischen Akteure (Walper, Müller, und Kleinschrot 2019).

Als eine «wichtige Voraussetzung für den Aufbau vertrauensvoller Beziehungen» wird im Beschluss der KMK die Etablierung weiterer «Kontaktmöglichkeiten, auch auf elektronischem Wege» benannt (KMK 2018a, 6). Abgesehen davon werden zur Zusammenarbeit von Eltern und Schule jedoch keine Empfehlungen zur digital unterstützten Kommunikation und Zusammenarbeit gegeben (KMK 2018b). In dem bereits zitierten Strategiepapier der Kultusministerkonferenz zur «Bildung in der digitalen Welt» (2016) wird jedoch das Potenzial hervorgehoben, das der Digitalisierung in Bezug auf den Informationsfluss und die Vernetzung zwischen Elternhaus und Schule sowie die Teilhabe von Eltern am schulischen Leben zukommt.

\section{Effekte der Kommunikation und Kooperation zwischen Elternhaus und Schule}

\subsection{1 Bedeutung und Begriffsbestimmung}

Die Kommunikation und Kooperation von Eltern und Schule ist von zentraler Bedeutung für die akademische und soziale Entwicklung von Schülerinnen und Schülern und insgesamt für die Erfüllung des schulischen Leistungsauftrags (Boonk u. a. 2018; Otterpohl und Wild 2017). Die Quantität und Qualität der Kommunikations- und Kooperationsprozesse kann in diesem Zusammenhang die Beteiligung von Eltern an den Lernprozessen der Schülerinnen und Schüler verbessern und so auch das Engagement und die Leistungsentwicklung dieser positiv beeinflussen (Boonk u. a. 2018; Kraft und Dougherty 2013; Smith u. a. 2020). 
Beteiligungsprozesse umfassen sowohl das häusliche Engagement (z. B. Unterstützung der Hausaufgaben; Gespräche über schulische Erfahrungen) als auch das schulische (z. B. Kommunikation mit Lehrkräften; Übernahme von schulischen Aufgaben). Eines der am häufigsten angeführten Modelle zur Systematisierung der Elternbeteiligung wurde von Joyce Epstein (1995; Epstein u. a. 2018) vorgelegt und unterscheidet sechs Arten des «Parental Involvement»: (1) Elterliche Fürsorge und Erziehung, (2) Unterstützung des Lernens zu Hause, (3) Kommunikation mit der Schule, (4) Teilnahme an freiwilligen bzw. ehrenamtlichen Tätigkeiten, (5) Mitarbeit in Gremien und (6) Zusammenarbeit mit ausserschulischen öffentlichen Einrichtungen. Dabei nimmt die Kommunikation eine zentrale Stellung ein, da sie die erfolgreiche Zusammenarbeit zwischen Eltern und Lehrkräften erst ermöglicht (Bouffard 2006). Kommunikationsprozesse umschliessen regelmässige Gespräche zum Leistungsstand des Kindes, zu Möglichkeiten der Leistungsentwicklung, zur Schulwahl, Informationen zu Unterrichtsinhalten sowie zu weiteren Aktivitäten im Bereich der Schule (Epstein u. a. 2018)

Die Beteiligung von Eltern findet jedoch nicht in allen Bereichen von Schule mit gleicher Häufigkeit statt: Formen der Beteiligung, die die direkte Unterstützung des eigenen Kindes betreffen, treten am häufigsten auf, insbesondere die Teilnahme an Beratungsgesprächen mit Lehrkräften (Hertel, Jude, und Sälzer 2017). Schulbezogene Formen des Engagements, wie etwa die Unterstützung bei Exkursionen, sind demgegenüber deutlich seltener (ebd.). Ausserdem scheinen die verschiedenen Formen der Elternbeteiligung nicht im gleichen Umfang einen Einfluss auf die akademische Entwicklung der Schülerinnen und Schüler zu haben. Boonk u. a. (2018) weisen beispielsweise darauf hin, dass vor allem direkte Formen der Unterstützung wie die Kommunikation mit den Eltern über Lernaktivitäten im Schulalltag und insbesondere deren Ermutigung und Unterstützungsleistungen beim Lernen positive Effekte haben. Dies wird auch durch eine Metaanalyse von Smith u. a. (2020) unterstützt, in der die Autorinnen und Autoren zeigen konnten, dass insbesondere das Engagement der Eltern in den häuslichen Lernprozessen der Schülerinnen und Schüler effektiv für die akademische und sozio-emotionale Entwicklung der Kinder ist.

Die grosse Bedeutung einer Beteiligung von Eltern an der schulischen Entwicklung ihrer Kinder konnte im Rahmen zahlreicher Überblicksarbeiten und Metaanalysen der empirischen Bildungsforschung bestätigt werden (Boonk u. a. 2018; Park und Holloway 2017; Pekrun 2001). Auch Hattie (2009) benannte auf Basis seiner zusammenfassenden Auswertung von über 800 Metaanalysen das Involvement der Eltern als einen zentralen Einflussfaktor für den Schulerfolg der Kinder. Dieses Involvement umschliesst auch hier vor allem direkte, kindbezogene Formen, etwa dass Eltern mit ihrem Kind über schulische Belange kommunizieren, Interesse an Schulinhalten und Hausaufgaben zeigen und bei letzteren unterstützen, hohe Bildungsaspirationen und Leistungserwartungen äussern und mit dem Kind über seine schulische Entwicklung 
diskutieren (ebd.). Dabei scheint das Engagement sowohl kurz- als auch langfristig von Bedeutung: Kurzfristig fördern Eltern vor allem dann, wenn Probleme auftreten, langfristig sind die Effekte einer Förderung in der Grundschulzeit noch in der 9. Klasse nachweisbar (Hertel, Jude, und Sälzer 2017).

Von Bedeutung scheint in diesem Zusammenhang die Qualität und nicht die Quantität des elterlichen Involvements zu sein (Wild und Lorenz 2010). Zudem zeigen sich in zahlreichen Studien auch indirekte Effekte einer verbesserten Kommunikation und Kooperation zwischen Eltern und Lehrpersonen auf die schulischen Leistungen der Schülerinnen und Schüler: So kann ein stärkeres schulbezogenes Engagement von Eltern zu einer positiveren Einstellung von Schülerinnen und Schülern zur Schule, einer höheren Motivation und Aufmerksamkeit im Unterricht, einer erhöhten Wahrscheinlichkeit der Anfertigung von Hausaufgaben, einer Verbesserung der Beziehung zu Mitschülerinnen und -schülern bzw. Lehrenden sowie einer Reduktion von Fehlzeiten und Verhaltensauffälligkeiten führen (Desforges und Abouchaar 2003; Kraft und Dougherty 2013; Neuenschwander u. a. 2004). Allerdings sind die Ergebnisse empirischer Studien zum Zusammenhang zwischen schulbezogenen Formen der Elternbeteiligung und der Leistungsentwicklung weniger eindeutig als jene zur Beteiligung von Eltern an den Lernaktivitäten ihrer Kinder (Boonk u. a. 2018).

Weiterhin umfassen die Forschungsergebnisse auch heterogene Befunde. Beispielsweise kann eine Hausaufgabenbetreuung durch Eltern auch als aufdringlich und kontrollierend erlebt werden und ist dann mit entsprechend negativen Effekten verbunden (Boonk u. a. 2018). Gleiches gilt für Bildungsaspiration und Leistungserwartungen, die etwa mit Druck, Kontrolle, Einmischung und Strafen verbunden sind (Boonk u. a. 2018; Dumont u. a. 2014; Fan und Williams 2010; Lorenz und Wild 2007). Darüber hinaus können bestimmte Formen der Kommunikation zwischen Lehrpersonen und Eltern auch Barrieren professionellen und privaten Handelns überschreiten und zu einer vermehrten Einmischung in schulische oder private Belange führen, die sich ungünstig auf die Lehrperson-Eltern-Beziehungen und nachfolgend möglicherweise auch auf die schulische Entwicklung auswirken kann (Wasserman und Zwebner 2017).

\subsection{Veränderungen durch digitale Medien}

Neben den herkömmlichen Kommunikationswegen zeigt sich an Schulen eine zunehmende Nutzung digitaler Kommunikationsmittel. Dies betrifft vor allen Dingen die Nutzung von E-Mails, zunehmend aber auch die Nutzung von Social Media Apps sowie spezifische Anwendungen auf Online- bzw. Schulplattformen, in Lernmanagementsystemen und weitere Anwendungen auf mobilen Geräten für Textnachrichten und Telefonie (Thompson, Mazer, und Flood-Grady 2015). Im Rahmen einer Studie aus den USA zeigte sich, dass E-Mails als Kommunikationskanal von Eltern mehr als 
doppelt so häufig genutzt wurden wie Face-to-Face-Gespräche (ebd.). Welche computerunterstützten Kommunikationsformen im deutschsprachigen Raum mit welcher Häufigkeit im Allgemeinen genutzt werden, ist weitgehend unbekannt. Breiter und Ruhe (2018) haben in einer vergleichenden Studie die kommunikativen Praktiken von Schulen in England und Deutschland untersucht. Dabei zeigten sich auch charakteristische Unterschiede, wie beispielsweise die deutliche höhere Nutzung von sozialen Medien in den englischen Schulen und die deutlich geringere Einbindung von Lernmanagementsystemen in die Kommunikationsbeziehungen zwischen Elternhaus und Schulen in den deutschen Schulen. Aktuelle Untersuchungen zur coronabedingten Fernlehre (Huber u. a. 2020; Tengler, Schrammel, und Brandhofer 2020) lassen vermuten, dass auch hier der Grossteil der Kommunikation per E-Mail erfolgte, der Nutzung von Lernplattformen bzw. Lernmanagementsystemen aber eine zunehmende Rolle zukommt (ebd.).

Auch zu den Effekten einer digital unterstützten Kommunikation und Kooperation existieren nur wenige Forschungsergebnisse, die zudem sehr unterschiedliche Medien (Smartphone, Tablet etc.), Anwendungen (E-Mail, WhatsApp, LMS etc.) und mediale Praktiken mit sehr unterschiedlicher Zielsetzung (Information, Partizipation, Kooperation) untersuchen. Generell deuten erste Studien in diesem Bereich darauf hin, dass digitale Medien zu einer Verbesserung der Kommunikations- und Kooperationsprozesse zwischen Elternhaus und Schule führen können und somit zumindest den subjektiven Beobachtungen der beteiligten schulischen Akteure (Eltern, Lehrpersonen) zufolge - auch mit einer Förderung der schulischen Lernprozesse von Schülerinnen und Schülern einhergehen (Bouffard 2008; Olmstead 2013; Palts und Kalmus 2015; Thompson 2008; Thompson, Mazer, und Flood-Grady 2015). Darüber hinaus gibt es Hinweise auf eine Stärkung der partnerschaftlichen Zusammenarbeit durch die Nutzung digitaler Kommunikationsmittel (Kuusimäki, UusitaloMalmivaara, und Tirri 2019; Thompson 2008). Die Nutzung digitaler Medien kann beispielsweise die Frequenz und Effektivität der Kommunikation zwischen Eltern und Lehrkräften erhöhen, unter anderem bedingt durch die verbesserte Erreichbarkeit beider Seiten sowie die Einfachheit und Unmittelbarkeit der Kommunikation (Thompson 2008). Eltern und Lehrpersonen schätzen dabei vor allem auch asynchrone Kommunikationsformen für komplexe Nachrichten, bei denen der Inhalt und die Form der Nachricht überdacht und auf die Kommunikationssituation angepasst werden können (Thompson u. a. 2015). Die meisten Eltern und zu einem geringeren Anteil auch die Lehrkräfte befürworten die Möglichkeit einer Interaktion, die nicht die Präsenz von Eltern in der Schule erfordert (Olmstead 2013). Gleichwohl liegen Forschungsergebnisse vor, die zeigen, dass Eltern eine Face-to-Face-Kommunikation für bestimmte Gesprächsthemen bevorzugen - etwa, um Probleme zu klären (Palts und Kalmus 2015). 
Die Befunde deuten auch auf Nachteile einer digital unterstützten Kommunikation und Kooperation hin. Es zeigen sich Hinweise, dass die Nutzung digitaler Kommunikationsmittel die persönliche Kommunikation und den Austausch von Informationen auch behindern kann (Olmstead 2013; Wasserman und Zwebner 2017). Dies ist unter anderem abhängig von der Nutzungskompetenz, aber auch von den Einstellungen und Ängsten der Nutzenden, etwa davor, persönliche Informationen preiszugeben und damit digitale Spuren im Internet zu hinterlassen (Palts und Kalmus 2015). Sind mit der digital vermittelten Kommunikation Ängste verknüpft, kann sich dies nicht nur auf die Zusammenarbeit zwischen Elternhaus und Lehrpersonen, sondern auch auf deren Beziehung auswirken (Lee u. a. 2011; Olmstead 2013).

Weiterhin konnte in Fallstudien (Selwyn u. a. 2011) gezeigt werden, dass auch die digital vermittelte Kommunikation nicht immer wechselseitig ist, wie es für eine produktive Zusammenarbeit idealerweise der Fall sein sollte. Vielfach besteht auch hier die Zusammenarbeit nur in der einseitigen Distribution von Informationen (ebd.). Die digital unterstützte Kommunikation muss sich also dahingehend nicht von herkömmlicher Kommunikation zwischen Eltern und Lehrkräften unterscheiden (Lewin und Luckin 2010) und führt auch nicht automatisch zu einer Verbesserung. Epstein u. a. (2018) und Selwyn u. a. (2011) schlussfolgern aus den Ergebnissen ihrer Fallstudien, dass bestehende positive und negative Kommunikations- und Kooperationskulturen durch digitale Medien oft reproduziert oder sogar verstärkt werden.

\section{Faktoren für eine (gelingende) Elternbeteiligung}

Ob eine Elternbeteiligung gelingt und positive Effekte zeigt oder ob diesbezüglich Barrieren existieren, hängt von einer Vielzahl an Faktoren ab. Ein Modell zur Elternbeteiligung von Green u. a. (2007) fokussiert auf Gelingensbedingungen und ordnet diese drei Bereichen zu: (1) Motivationalen Überzeugungen der Eltern (Rollenverständnis und Selbstwirksamkeit), (2) der Wahrnehmung, ob das eigene Engagement willkommen ist (bei Schule, Lehrkraft und Kind), und (3) den Lebensumständen der Familie (eigene Fähigkeiten und Wissen, verfügbare Zeit und Energie). Hornby und Lafaele (2011) unterscheiden ebenfalls Faktoren, die eine Elternbeteiligung beeinflussen, gehen dabei aber über die Ebene der Eltern deutlich hinaus. Sie unterscheiden vier Bereiche: (1) Faktoren auf Ebene der Eltern bzw. Familie, (2) Faktoren auf Eltern-Lehrkraft-Ebene, (3) gesellschaftliche Faktoren und (4) kindbezogene Faktoren. In Anlehnung an Hornby und Lafaele (2011) werden im Folgenden Faktoren für eine digital vermittelte Zusammenarbeit zwischen Eltern und Schule zusammengetragen und in drei Bereiche unterschieden: Die Ebene der Eltern, der Lehrperson und die schulischen Rahmenbedingungen. Mit Bezug auf Green u. a. (2007) werden dabei insbesondere motivationale Faktoren und die Kompetenzen der Akteure in den Blick genommen. Die Ebene des Kindes wird aufgrund der Fokussetzung an dieser 
Stelle aussen vorgelassen. Darüber hinaus wird für jeden dieser Bereiche reflektiert, inwiefern sich durch die Nutzung digitaler Medien Veränderungspotenziale ergeben könnten. Empirisch lassen sich diese allerdings bisher nicht immer belegen. Ziel ist es daher, potenzielle Forschungsbedarfe herauszustellen.

\subsection{Faktoren auf Ebene der Eltern}

\section{Allgemein}

Ein zentraler Faktor für die Beteiligung von Eltern an schulischen Belangen scheint deren diesbezügliche Einstellung zu sein: Das umfasst das Verständnis der eigenen Rolle im Bildungsprozess des Kindes, den Glauben an die eigene Fähigkeit, das Kind unterstützen zu können und die Einstellung zum und das Verständnis vom kindlichen Lernen (Hill und Tyson 2009; Hornby und Lafaele 2011). Forschungsarbeiten im deutschsprachigen Raum verdeutlichen, dass Beteiligungsmöglichkeiten insgesamt noch wenig wahrgenommen und die Zusammenarbeit zwischen Eltern und Lehrkräften vornehmlich dann initiiert bzw. verstärkt wird, wenn sich Konflikte anbahnen oder bereits bestehen (Börner u. a. 2011; Hertel, Jude, und Naumann 2010; Sacher 2014; Wild und Lorenz 2010). Eine Studie von Sacher (2004; 2005) zeigt auf, dass Eltern an einer Zusammenarbeit vorwiegend dann interessiert sind, wenn die Belange und der Schulerfolg ihres eigenen Kindes im Mittelpunkt stehen. Während die befragten Eltern einer Unterrichts- und Bildungskooperation gegenüber offen sind und sich eine Beratung durch Lehrkräfte im Hinblick auf die Unterstützung der schulischen Entwicklung des Kindes wünschen, lehnt die Mehrheit eine Beratung im Hinblick auf die Erziehung des Kindes ab (ebd.; Sacher 2014). Ein bedeutsamer Faktor für die Beteiligung ist darüber hinaus die Wahrnehmung von Eltern, dass ihr Engagement willkommen ist - sowohl von Seiten der Lehrkraft als auch von Seiten des eigenen Kindes (Green u. a. 2007) - sowie die erlebte Selbstwirksamkeit bezüglich des eigenen Engagements (Green u. a. 2007; Yotyodying und Wild 2014). Schliesslich können auch wahrgenommene Defizite des Kindes zu einer Verstärkung des Engagements führen (z. B. Dumont u. a. 2014).

Auch die Lebensumstände der Familie nehmen einen Einfluss auf die Elternbeteiligung. Beispielsweise können die Arbeitssituation und eine dadurch bedingte geringere Flexibilität bzw. der Mangel an Zeit aufgrund anderweitiger Verpflichtungen das Engagement von Eltern beeinflussen (Epstein u. a. 2018; Hall u. a. 2005; Lee und Bowen 2006). Dies schlägt sich auch in der Wahrnehmung der Eltern bezüglich der verfügbaren Zeit und Energie nieder, welche sich wiederum nachweislich auf das Engagement von Eltern auswirkt (Green u. a. 2007). 
Weiterhin spielen das elterliche Bildungsniveau und der sozio-ökonomische Status in diesem Zusammenhang eine Rolle. Das Bildungsniveau hängt beispielsweise eng mit den Bildungsaspirationen und Leistungserwartungen an die Kinder sowie einer höheren Motivation zusammen, sich für diese Ziele zu engagieren (Boonk u. a. 2018). Forschungsergebnisse verdeutlichen, dass Eltern mit einem Hochschulabschluss sich häufiger im Schulkontext engagieren, häufiger Bildungsdiskussionen mit dem Kind führen und höhere Bildungserwartungen haben (Lee und Bowen 2006). Weiterhin schätzen Eltern mit geringerem Bildungsniveau ihre Kenntnisse und ihr Wissen für eine Beteiligung öfter als nicht ausreichend ein (Green u. a. 2007). Eine generell geringere Beteiligung von Eltern aus Familien mit niedrigem Bildungsniveau lässt sich jedoch pauschal nicht nachweisen. Ergebnisse zum sozio-ökonomischen Status sind oft mit dem Bildungsniveau der Eltern konfundiert, wobei das Bildungsniveau hier der entscheidende Faktor zu sein scheint (Boonk u. a. 2018).

Ähnliches gilt in Bezug auf den unterschiedlichen ethnischen und kulturellen Hintergrund von Eltern. Studien zeigen hier keine generell geringere Elternbeteiligung bzw. Qualität der Kommunikation (Murray, McFarland-Piazza, und Harrison 2015; Schu 2014). Auch hier sind es insbesondere das Bildungsniveau der Eltern und die damit zusammenhängenden Bildungserwartungen, welche die Elternbeteiligung beeinflussen. Es gibt jedoch Hinweise darauf, dass sich Familien mit Migrationshintergrund nicht immer als gleichberechtigte Partner wahrnehmen und sich bei Leistungsschwierigkeiten oft nicht unterstützt bzw. in einer Bringschuld fühlen (Hawighorst 2009). Ausserdem erschweren Sprachbarrieren hier des Öfteren die Kommunikation mit der Schule (s. folgender Abschnitt).

Ein eng mit dem Bildungsniveau und dem sozio-ökonomischen Hintergrund in Verbindung stehender Faktor für die Elternbeteiligung ist die Kenntnis der spezifischen Sprache und Normen der Schule. Dies betrifft beispielsweise die umgangssprachliche Verwendung bestimmter Fachtermini oder Verweise auf pädagogische Theorien, aber auch stärker formalisierte Sprachstrukturen, die erst durch entsprechende Erfahrungen in diversen Bildungskontexten verständlicher und vertrauter werden. Die mangelnde Kenntnis und die mangelnde Erfahrung im Umgang mit der spezifischen Sprache und den Normen der Schule kann ein Hindernis für die Beteiligung von Eltern darstellen (Hattie 2009). Dabei sehen sich insbesondere Eltern mit niedrigem sozio-ökonomischem Status mit Sprachpraktiken und Normen konfrontiert, die ihnen nicht unbedingt vertraut sind (ebd.). Im Rahmen des fünfjährigen «Flaxmere»-Projekts (Clinton, Hattie, und Dixon 2007) - einer Evaluation von fünf Schulen in Neuseeland mit dem niedrigsten sozio-ökonomischen Status - war die Verbesserung der Elternhaus-Schule-Beziehung ein wichtiges Projektziel. Dazu wurden Eltern mit einem niedrigen sozio-ökonomischen Hintergrund mit der Sprache von Schule, Lehr-Lern-Kontexten und einer angemessenen Hilfestellung für ihr Kind 
vertraut gemacht. Dies führte nachweislich zu einem stärkeren Involvement in die Bildungsprozesse ihrer Kinder und einem verstärkten freiwilligen Engagement im schulischen Kontext.

Ein weiterer gut nachgewiesener Zusammenhang ist der zwischen Geschlecht und Elternbeteiligung. Hier zeigten Forschungsergebnisse wiederholt auf, dass sich verstärkt Mütter im schulischen Kontext engagieren (Jeynes 2005), bzw. die Beteiligung insgesamt sinkt, wenn Mütter berufstätig sind (Castro u. a. 2004).

\section{Digital unterstützte Kommunikation und Kooperation}

Ob eine digital vermittelte Kommunikation bestimmte Kommunikationsbarrieren zwischen Eltern und Schule senkt, die Einstellung von Eltern zur Zusammenarbeit und zu schulischen Fragen verändert oder gar das Verständnis ihrer Rolle im Bildungsprozess des Kindes beeinflusst, ist bisher weitgehend unerforscht.

Erste Studien zu den personalen Bedingungen der Nutzung digitaler Medien zur Kommunikation auf Seite der Eltern (Macia Bordalba und Garreta Bochaca 2019) zeigen, dass die Nutzung dieser vor allem von den Vorstellungen zu den Wirkungen auf den entsprechenden sozialen Kontext und weniger von den spezifisch medienbezogenen Überzeugungen beeinflusst werden. So sind viele Eltern in ihren medienbezogenen Überzeugungen durchaus positiv eingestellt und sehen hier das Potenzial digitaler Medien in Bezug auf die Schnelligkeit, Spontaneität und Praktikabilität der Kommunikation (Macia Bordalba und Garreta Bochaca 2019; Thompson, Mazer, und Flood-Grady 2015). Bedenken richten sich vor allem auf den sozialen Kontext. So wird befürchtet, dass sich Nachteile in der Kommunikation zu komplexeren und ernsthafteren Themen ergeben oder die Qualität der persönlichen Beziehungen mit der Lehrperson durch die Abwesenheit nonverbaler Informationen leiden könnte (ebd.). Manche Eltern präferieren deswegen soziale Medien wie Facebook und IP-Telefonie für die Kommunikation mit Lehrkräften und benennen Vorteile wie die Möglichkeit eines direkten Feedbacks oder die Präferenz akustischer bzw. visueller Formate (Thompson, Mazer, und Flood-Grady 2015). Macia Bordalba und Garreta Bochaca (2019) betonen, dass eine gelingende digitale Kommunikation und Kooperation eine gute Passung der entsprechenden Einstellungen von Eltern und Lehrpersonen voraussetzt.

Es zeigen sich weiterhin Hinweise darauf, dass eine digital unterstützte Kommunikation und Kollaboration eine hinderliche Lebens- und Arbeitssituation zumindest teilweise kompensieren und hier zu positiven Effekten für die Zusammenarbeit zwischen Elternhaus und Schule führen kann (Thompson, Mazer, und Flood-Grady 2015). So lässt sich z. B. eine geringere zeitliche Flexibilität durch eine digitale, zeit- und ortsunabhängige Elternbeteiligung ausgleichen. Dies kann insbesondere für Mütter oder Väter mit starker beruflicher und familiärer Beanspruchung und für Familien mit geringerem sozio-ökonomischen Status entlastend sein. Mehrere 
Studien verdeutlichen, dass es vor allem auch solche Gründe sind, weshalb Eltern eine Kommunikation mit Lehrpersonen via E-Mail oder Chat bevorzugen (Natale und Lubniewski 2018; Thompson, Mazer, und Flood-Grady 2015). Dies liegt insbesondere in den allgemeinen Vorteilen asynchroner Kommunikation begründet - etwa der Möglichkeit, Nachrichten zu einem Zeitpunkt der eigenen Wahl lesen und beantworten zu können.

Ob Eltern aus Familien mit geringerem Bildungsniveau oder mit kulturell bzw. sprachlich unterschiedlichem Hintergrund durch eine digitale Kommunikation und Kooperation besser erreicht werden können, wurde bisher noch nicht umfassend untersucht. Theoretisch lassen sich hier unterschiedliche Effekte vermuten. Zum einen könnten digitale Medien bestimmte Sprachbarrieren reduzieren und so eine Kommunikation zwischen Eltern und Schule erleichtern. Beispielsweise könnte eine digital vermittelte Kommunikation insofern Potenziale bergen, als dass niedrigschwelliger mit Übersetzungen oder Begriffsklärungen gearbeitet werden kann (z. B. Fremdsprache, akademische Sprache). Eine Studie zur Präferenz von Kommunikationskanälen zeigt etwa eine Bevorzugung der E-Mail-Kommunikation gegenüber der Face-toFace-Kommunikation von Eltern. Grund dafür ist unter anderem die Möglichkeit, Informationen, eigene Gedanken und Rückmeldungen ohne Zeitdruck formulieren und besser erläutern zu können (Thompson, Mazer, und Flood-Grady 2015). Eine Vertrautheit mit digitalen Medien oder bestimmten Anwendungen könnte sich hier unterstützend auswirken, da diese gegebenenfalls die Nutzung von Anwendungen zur Übersetzung oder Korrektur erleichtern.

Zum anderen erfordern komplexere digitale Nutzungsszenarien eine entsprechende Medienkompetenz auch auf Seiten der Eltern, die weit über einfache Anwendungskompetenzen hinausgeht. Dies wird beispielsweise dann relevant, wenn die Kommunikationspraktiken in einer Schule, bedingt durch die unterschiedlichen Nutzungspraktiken der Lehrpersonen, sehr divers sind. Ein weiteres Beispiel ist die Verfügbarmachung und Nutzung schulbezogener Daten und Informationen für Eltern auf Onlineplattformen bzw. in Lernmanagementsystemen. Solche Daten können unter Umständen regelmässige und fortlaufende Berichte über die Unterrichtsaktivitäten und -leistungen der Schülerinnen und Schüler umfassen und haben damit weitreichende Konsequenzen für die Ausgestaltung und die Überwachung schulischer Prozesse und Aktivitäten (Manolev, Sullivan, und Slee 2019). Hier besteht möglicherweise auch die Gefahr, dass digital unterstützte Kommunikations- und Kooperationsformen zu einer Benachteiligung bestimmter Elterngruppen, z. B. mit einem niedrigeren sozio-ökonomischen Hintergrund, führen könnten. Dies ist ein wichtiger Punkt für die zukünftige Forschung.

Ein geschlechtsspezifischer Zusammenhang zwischen der Nutzung digitaler Medien für die Zusammenarbeit zwischen Elternhaus und Schule und der Beteiligung von Müttern und Vätern an den Bildungsprozessen ihrer Kinder lässt sich bisher nicht 
nachweisen: Eine Studie zur Veränderung einer Beteiligung von Vätern an schulischen Belangen hat nicht den erwünschten Effekt gezeigt, sondern das Ungleichgewicht zugunsten von Frauen blieb auch bei Nutzung digitaler Kommunikationssysteme bestehen (Blau und Hameiri 2012; 2017). Allerdings existieren auch hier keine differenzierten Forschungsergebnisse zu unterschiedlichen digitalen Medien und Kommunikations- bzw. Kooperationsanlässen.

Insgesamt zeigen sich also zahlreiche Forschungsbedarfe sowohl bezogen auf die Überzeugungen und Kompetenzen von Eltern als auch im Hinblick auf eine sprachlich, kulturell und sozio-ökonomisch diverse Elternschaft. Hier sollten in einem ersten Schritt Fragen geklärt werden, inwieweit sich Veränderungen ergeben, wer (mit welchem sozio-ökonomischen Hintergrund, Geschlecht bspw.), wie (in welcher Qualität der Anfragen, auf welchen Kommunikationswegen), worüber (mit welchen Interessen und Anliegen) und in welcher Form (Frequenz, zeitliche Rhythmen) kommuniziert. Wie interagieren digitale Medien mit den verschiedenen bereits in der Forschung benannten Einflussfaktoren einer gelingenden Kommunikation? Wie könnte die digital unterstützte Kommunikation gestaltet werden, um die Beteiligung von Eltern unterschiedlicher sozio-ökonomischer Hintergründe, Ethnien und Migrationshintergründe zu erhöhen?

\subsection{Faktoren auf Ebene der Lehrperson}

\section{Allgemein}

Weitere Faktoren für die Elternbeteiligung lassen sich auf der Ebene der Lehrperson bzw. auf der Beziehungsebene zwischen Eltern und Lehrkräften verorten. Beispielsweise können unterschiedlich gelagerte Vorstellungen und Überzeugungen zu Verantwortlichkeiten von Eltern und Lehrkräften oder darüber, was eine «gute» Zusammenarbeit ausmacht, in diesem Zusammenhang zu Barrieren für die Elternbeteiligung führen (Hornby und Lafaele 2011). Eine Studie von Sacher (2008) verdeutlicht, dass Lehrkräfte grösstenteils die Eltern in der Verantwortlichkeit sehen, Informationen über schulische Belange einzuholen (z. B. über das Arbeitsverhalten des eigenen Kindes) und gleichzeitig davon ausgehen, dass Eltern in der Pflicht stehen, die Schule über familiäre Belange zu informieren (z. B. über Veränderungen im Familienleben, die sich auf die Leistungen auswirken könnten). Dies wird durch entsprechende Studien zur Beratungspraxis im Schulalltag unterstützt, die eine gewisse Zurückhaltung auf Seiten der Lehrpersonen hinsichtlich des Angebots von Elternberatung erkennen lassen (Hertel, Bruder, und Schmitz 2009).

Unterschiedliche Vorstellungen existieren nicht nur zu den Verantwortlichkeiten, sondern auch zu den Zielen und Inhalten der Kommunikation zwischen Eltern und Lehrkräften, die sich ebenfalls auf die Qualität der Zusammenarbeit zwischen 
Elternhaus und Schule auswirken können. Es wurde bereits darauf hingewiesen, dass für Eltern das vorrangige Ziel der Kommunikation meist in einem Informationsaustausch und der pädagogischen Beratung im Hinblick auf die schulische Situation der Schülerin oder des Schülers besteht (Hertzsch und Schneider 2018). Oft sind es aber die existierenden oder potenziellen Schwierigkeiten und Konflikte, die den Kommunikationsanlass bilden. Entsprechend findet eine Kontaktaufnahme von Lehrendenseite zwecks Lobs oder positiver Nachrichten nur selten statt (Penney u. a. 2019). Gerade aber die Rückmeldung zu den Stärken des Kindes wird von Eltern als wichtig bewertet. So wünschen sich die Eltern, dass Lehrende auch «die Stärken kommentieren und eine ehrliche und offene Kommunikation fördern sollten» (ebd., 210).

Darüber hinaus stellen Kompetenzen zur Gesprächsführung eine wichtige Voraussetzung für Lehrpersonen dar, um erfolgreich mit Eltern zu kommunizieren und zu kooperieren (Aich u. a. 2017). Unterschiedliche Befragungen weisen nach, dass sich Lehrpersonen in Elterngesprächen häufig überfordert fühlen und die Ausbildung in diesem Bereich als nicht ausreichend beschrieben wird (Hertel 2009). Die Beratung und Beurteilung nimmt einen hohen Rang bei den als belastend eingestuften Tätigkeitsbereichen von Lehrpersonen ein (Aich u. a. 2017). Ein Grossteil der Lehrkräfte hat weder Kommunikationstechniken noch die Auseinandersetzung mit Eltern zugunsten des Beziehungsausbaus erlernt, was zu einem zentralen Hindernis für eine engere und effektive Kooperation werden kann (Hertzsch und Schneider 2018). Dementsprechend wird die Kommunikation von Elternseite teilweise als defizitär erlebt. Dies gilt beispielsweise für Familien aus «nicht-traditionellen» Familienstrukturen oder mit sozial und kulturell diversen Lebenszusammenhängen, die hier Kommunikationsbarrieren ausmachen (Dor 2018; Gomolla und Rotter 2014). In der Wahrnehmung der Eltern führt dies dazu, dass die Lehrpersonen weniger über die Kinder wissen, was ein Gefühl von Ausgeschlossenheit bewirken und damit die zukünftige Motivation zur Zusammenarbeit senken kann (ebd.). Studienergebnisse weisen darauf hin, dass das Engagement und die Motivation zur Beteiligung von Eltern deutlich erhöht werden können, wenn entsprechende Impulse von Lehrpersonen erfolgen (Hilkenmeier, Wiescholek, und Buhl 2017).

Neben der unterschiedlichen Gewichtung von Verantwortlichkeiten, den Überzeugungen zur Kommunikation und Kooperation mit Eltern und den Kompetenzen für eine Zusammenarbeit, lassen sich als Gründe für eine fehlende Information und Kooperation auch die verfügbare Zeit und fehlende Ressourcen auf Seiten der Lehrkraft anführen (Hertzsch und Dörflinger 2018). Dies wird begünstigt durch hohe Schülerzahlen und ungünstige schulorganisatorische Strukturen (s. Abschnitt 4.3 Faktoren auf Ebene der Schule). 
Digital unterstützte Kommunikation und Kooperation

Es stellt sich die Frage, inwieweit digital unterstützte Kommunikations- und Kooperationsprozesse bestimmte Barrieren hinsichtlich der Überzeugungen und Kompetenzen von Lehrpersonen sowie deren Belastung und Belastungsempfinden verändern. Beispielsweise könnte digital vermittelte Kommunikation durch die Flexibilität und den niedrigeren Aufwand bei der Organisation auch zu einem geringeren Zeitaufwand für die Beteiligten führen. Andererseits könnte es aufgrund der Vielfältigkeit digitaler Kommunikationswege und der leichteren Verfügbarkeit und Ansprechbarkeit von Lehrpersonen auch zu einer höheren Belastung für diese kommen. Forschungsergebnisse weisen darauf hin, dass die Kommunikation über digitale Medien einen Einfluss auf das Belastungsempfinden haben könnte (Blau und Hameiri 2017). Diese sind jedoch nicht ganz eindeutig: So nehmen Lehrkräfte die Nutzung digitaler Medien zur Kommunikation als schneller, komfortabler und effizienter wahr (Blau und Hameiri 2017; Olmstead 2013; Palts und Kalmus 2015; Wasserman und Zwebner 2017). Trotzdem stellt der (zusätzliche) Versand von digitalen Nachrichten auch eine erhöhte Arbeitsbelastung dar und ist für die Lehrenden sehr zeitintensiv (Palts und Kalmus 2015). Ein höherer Zeitaufwand ergibt sich unter anderem durch die Bemühung, möglichst präzise und objektive Formulierungen zum Leistungsstand oder Verhalten des Kindes zu finden - bedingt durch die Angst vor einem «digital footprint» und damit der bleibenden Verfügbarkeit der digitalen Nachricht im Netz (ebd.). Darüber hinaus entstehen zusätzliche Belastungen, die insbesondere im Zuge der COVID-19 Pandemie deutlich geworden sind (Eickelmann und Drossel 2020; Huber u. a. 2020), beispielsweise die Notwendigkeit einer parallelen Kommunikation mit Eltern und Lernenden in Zeiten des Wechselunterrichts, der in Phasen in der Schule und in Phasen zu Hause stattfindet.

Ob Lehrpersonen die neuen Potenziale sehen und nutzen können und ob sich Effekte auf die Vielfalt und Inhalte der Kommunikation ergeben, muss vertiefend untersucht werden. Es gibt Hinweise darauf, dass Eltern und Lehrkräfte ihre Kommunikationskanäle abhängig von Zielen und Inhalten der Kommunikation wählen: Für persönliche Themen bzw. Gespräche über Probleme oder Konflikte wird die Face-toFace-Kommunikation bevorzugt (Bouffard 2008; Thompson 2008), während digitale Medien zur Vermittlung allgemeiner Informationen genutzt werden (Bouffard 2008). Allerdings zeigen die Ergebnisse von Thompson u. a. (2015) auch, dass eine solche Kategorisierung im Sinne der Media Richness Theory (Daft und Lengel 1986) zu kurz greift, da auch für konfliktreiche Situationen sowohl von Eltern als auch Lehrpersonen medial einfachere und asynchrone Kommunikationskanäle bewusst und gezielt ausgewählt werden.

Ebenso wie analoge Kompetenzen zur Gesprächsführung werden auch digitale Kommunikationskompetenzen bisher im Rahmen der Lehrerausbildung und -weiterbildung nicht ausreichend vermittelt. Dadurch werden die Potenziale zu wenig 
genutzt und es können sich Missverständnisse zwischen Eltern und Lehrkräften ergeben (Palts und Kalmus 2015; Alanko 2018). Dies kann dazu führen, dass traditionelle Wege der Kommunikation bevorzugt werden (Palts und Kalmus 2015). Selbst in einigen Ländern, wie etwa Finnland oder Estland, in denen eine digitale Kommunikation zwischen Eltern und Lehrkräften etabliert ist und persönliche Treffen stellenweise selten sind, zeigen sich zum Teil Vorbehalte (Kuusimäki, Uusitalo-Malmivaara, und Tirri 2019; Palts und Kalmus 2015).

Darüber hinaus stellt sich die Frage, welche Kompetenzen Lehrpersonen zur Nutzung digitaler Medien als Kommunikations- und Kooperationsmittel haben. Nur ein Viertel der deutschen Lehrpersonen gibt an, im Rahmen der Lehramtsausbildung generell den Umgang mit digitalen Medien erlernt zu haben - darunter ein signifikant geringerer Prozentsatz an weiblichen Lehrkräften (Drossel u. a. 2019). Ein noch niedrigerer Anteil an Lehrkräften (etwa ein Drittel) gibt an, ein Lernmanagementsystem benutzen zu können, womit Deutschland deutlich unterhalb des internationalen Mittelwertes liegt. Insbesondere jüngere und männliche Lehrkräfte geben hier höhere Kompetenzen an. Auch zeigen sich Schulformunterschiede: An Gymnasien ist der Anteil höher im Vergleich zu anderen Schulformen (ebd.).

Ob digitale Medien überhaupt im schulischen Kontext eingesetzt werden, hängt ausserdem davon ab, welche Vor- und Nachteile Lehrende für ihre Arbeit sehen (Ertmer, Ottenbreit-Leftwich und Tondeur 2015; Prasse, Döbeli Honegger, und Petko 2017). In der vor allem im angloamerikanischen Raum populären Unified Theory of Acceptance and Use of Technology wird betont, dass die Nutzung und Akzeptanz einer neuen Technologie u. a. mit deren Relevanz für das berufliche Aufgabenfeld zusammenhängt (Venkatesh u. a. 2003; Venkatesh, Thong, und Xu 2012). Zahlreiche Studien konnten einen Zusammenhang zwischen einer positiven Einstellung von Lehrpersonen zum Einsatz digitaler Medien und deren tatsächlicher Nutzung in Schule und Unterricht empirisch nachweisen (Petko 2012; Prasse 2012). Auch in Bezug auf die digital unterstützte Kommunikation und Kooperation zeigen sich Hinweise, dass die Nutzungskompetenz, aber auch die Einstellungen und Ängste von Lehrpersonen einen Einfluss darauf haben, inwieweit diese für die Zusammenarbeit genutzt wird (Palts und Kalmus 2015). Typische Ängste richten sich beispielsweise darauf, nachzuverfolgende und auch für Eltern sichtbare «digitale Spuren» zu hinterlassen, oder auch auf eine Verletzung etablierter Kommunikationsstrukturen - etwa eine informellere Form und eine eventuell dadurch bedingte Missachtung der professionellen Rolle der Lehrperson vor allem bei Nutzung mobiler Technologien (z. B. Wasserman und Zwebner 2017). Insgesamt ist es also bedeutsam, die wahrgenommenen Vorund Nachteile einer digital unterstützten Kommunikation auch in der Forschung zukünftig stärker in den Blick zu nehmen. 
Weiterhin ist möglicherweise auch das Alter der Lehrperson ein Einflussfaktor auf die Bereitschaft, digitale Medien für die Kommunikation und Kooperation mit den Eltern zu nutzen. So haben Forschungsergebnisse zur allgemeinen Nutzung digitaler Medien im Unterricht gezeigt, dass ein höherer Anteil jüngerer Lehrkräfte digitale Medien in ihrem Unterricht höherfrequent (täglich und wöchentlich) nutzt (Drossel u. a. 2019). Allerdings zeigen sich solche Alterseffekte nicht konsistent über verschiedene Studien hinweg und hängen ausserdem nicht notwendigerweise mit der Qualität der Nutzung digitaler Medien im Unterricht zusammen (Prasse, 2012). Jüngere Lehrpersonen haben jedoch im Durchschnitt zu einem grösseren Anteil in ihrer Ausbildung entsprechende Fähigkeiten erworben (Drossel u. a. 2019). Es bleibt also auch hier zu prüfen, ob und wie weitere Faktoren, wie das Alter oder das Geschlecht, einen Einfluss auf die Nutzung digitaler Medien zur Kommunikation und Kooperation haben.

\subsection{Faktoren auf Ebene der Schule}

\section{Allgemein}

Darüber hinaus haben noch andere Rahmenbedingungen auf Ebene der Schule direkt oder indirekt einen Einfluss auf die Kommunikation und Kooperation zwischen Elternhaus und Schule (Sacher 2006). Dazu gehören zum einen institutionelle bzw. organisationale Strukturen und Abläufe, die sich zum Beispiel durch die gegebene Schulform, die Klientel der Schule (Zusammensetzung nach sozio-ökonomischem Status und Bildungsniveau), die Schülerzahl und Klassengrösse, verfügbare personale und finanzielle Ressourcen und auch rechtliche Rahmenbedingungen ergeben. So werden Klassengrössen und fehlende zeitliche Ressourcen von Lehrpersonen oft als Hinderungsgrund für eine optimal ausgestaltete Zusammenarbeit mit den Eltern beschrieben. Die Zusammensetzung der Elternschaft zeigt insbesondere über das vorhandene Bildungsniveau einen Zusammenhang mit dem schulischen Engagement der Eltern (s. Abschnitt Faktoren auf Ebene der Eltern). Eine Studie zum Vergleich der Elternbeteiligung in unterschiedlichen Schulformen und Schulstufen (Börner u. a. 2011) konnte darlegen, dass eine umfassende Elternbeteiligung im Primarbereich deutlich stärker ausgeprägt ist als im Sekundarbereich. Zudem existiert auf der Sekundarstufe in Gymnasien sowie Gesamtschulen eine stärkere Elternbeteiligung als in Haupt- und Realschulen (ebd.). Dies ging aber auch mit einem schlechteren Angebot an Beteiligungsmöglichkeiten an Haupt- und Realschulen einher (ebd.).

Auf einer schulkulturellen oder sozialen Ebene geht es um eine entsprechende Kooperations- und Führungskultur sowie das an der Schule existierende gemeinsame Verständnis und die Ziele hinsichtlich der Zusammenarbeit mit der Elternschaft. Diese werden vor allem auch von der Schulleitung und anderen zentralen Schlüsselakteuren ausgestaltet. So zeigte sich in der bereits erwähnten Studie von Börner 
u. a. (2011), dass in den Gesamtschulen der Elternbeteiligung auch insgesamt ein höherer Stellenwert beigemessen wurde. Intensive Massnahmen zur Schaffung von Gesprächsangeboten und eine starke Beziehungspflege scheinen hier im Gegensatz zu institutionellen Bedingungen einen grösseren Einfluss auf die die Elternbeteiligung zu haben (Stange u. a. 2012).

\section{Digital unterstützte Kommunikation und Kooperation}

Im Kontext der Nutzung und Integration digitaler Medien in schulische Abläufe und Prozesse allgemein konnten inzwischen zahlreiche Studien die Bedeutung der organisationalen Bedingungen einer Schule nachweisen (für einen Überblick s. Schaumburg und Prasse 2019, 246ff.). Auch in Bezug auf den speziellen Kontext einer digital unterstützten Kommunikation und Kooperation zwischen Elternhaus und Schule zeigen sich Hinweise, dass diese Bedingungen hier eine wichtige Rolle spielen.

Auf der Ebene institutioneller Strukturen bedarf eine digital optimal unterstützte Zusammenarbeit zwischen Elternhaus und Schule einer entsprechenden ICT-Infrastruktur, die sowohl die Verfügbarkeit mobiler digitaler Geräte für alle Beteiligten gewährleistet als auch ICT-Anwendungen umfasst, die an die Informations- und Kommunikationsbedürfnisse von Schule und Eltern (und Schüler/innen) angepasst sind. So war in einer Studie von Heath, Maghrabi und Carr (2015) die mangelnde Übereinstimmung in den ICT-bezogenen Präferenzen und Erwartungen zwischen Eltern und Schulleitung eine bedeutsame Barriere für die Implementation eines entsprechenden Online-Systems. Im Hinblick auf die komplexen Informations- und Kommunikationsprozesse, wie sie in Lern- und/oder Schulmanagementsystemen realisiert werden, sollte auch dem ICT-Support einer Schule als Schnittstelle zwischen den Bedürfnissen verschiedener Akteursgruppen eine zunehmend wichtige Rolle zukommen. Zudem stellen sowohl die rechtlichen als auch die in der Schule gegebenenfalls zusätzlich vereinbarten Bedingungen und Regeln zum Umgang mit den anfallenden (persönlichen) Daten einen sehr bedeutsamen Faktor für die Ausgestaltung der Kommunikations- und Kooperationsbeziehungen zwischen Elternhaus und Schule dar (Breiter, Lange, und Stauke 2008; Ifenthaler u. a. 2020; Prasse, Döbeli Honegger, und Hielscher 2021). Insbesondere die zunehmende und oft nicht von allen schulischen Akteuren umfassend reflektierte Nutzung von Lernmanagementsystemen stellt hier eine neue Herausforderung für Schule dar (Manolev, Sullivan, und Slee 2019).

Auf schulkultureller Ebene besteht die Frage, in welchem Ausmass eine existierende Kommunikations- und Kooperationskultur die digitale Zusammenarbeit prägt und wie sich diese umgekehrt durch die Besonderheiten digitaler Kommunikation auch verändern kann. Hierzu existiert bislang nur wenig Forschungsliteratur, die erwartungsgemäss in beide Richtungen weist. So deuten Studien darauf hin, dass digital umfassende Kommunikationspraktiken der Lehrpersonen einen positiven Einfluss 
auf die Elternbeteiligung haben (Blau und Hameiri 2017) und insbesondere spezielle Managementteams an Schulen sich positiv auf die Einführung neuer digital unterstützter Kommunikationsformen auswirken (Macia Bordalba und Garreta Bochaca 2019). Andererseits zeigen Breiter und Ruhe (2018) auf, wie unterschiedliche Vorstellungen und Praktiken zu einer datenbasierten Schulentwicklung weitreichende Konsequenzen für die Implementation unterschiedlicher Informations- und Kommunikationssysteme haben. Weiterhin deuten Studien darauf hin, dass bestehende Kontroll- und Effektivitätsbedürfnisse auf beiden Seiten - Elternhaus und Schule - oder gar die Aufrechterhaltung eines Machtstatus quo bestimmter Schulakteure auch zu einer ungünstigen Nutzung von Online-Informations- und Kommunikationssystemen führen kann (Perelman 2014; Selwyn u. a. 2011).

Insgesamt ist es also für die zukünftige Forschung bedeutsam, die Frage vertieft zu untersuchen, inwieweit die Kommunikation mit digitalen Medien zu Veränderungen der Kommunikations- und Kooperationskultur führt (etwa im Kontext der Ganztagsschule), inwieweit diese Veränderungen auch zu einer Verschiebung der Unterschiede zwischen verschiedenen Schulformen und Altersstufen führen und wie die Einführung neuer digital unterstützter Kommunikationsformen in den Schulen optimal begleitet und kritisch reflektiert werden sollte.

\section{Fazit und Forschungsagenda}

Wie die Ausführungen in den letzten Abschnitten gezeigt haben, wird eine Untersuchung und Diskussion der digital unterstützten Kommunikation und Kooperation zwischen Elternhaus und Schule in der bisherigen Forschungsliteratur noch weitgehend vernachlässigt. Es fehlt zum einen an einer grundlegenden Systematik der Bedingungen und Effekte einer effektiven digital unterstützten Kommunikation und Kooperation und zum anderen an empirischen Studien, die diese verschiedenen Bedingungen und Effekte in den Blick nehmen und die Konsequenzen für die Elternbeteiligung wie auch die schulischen Kommunikations- und Kooperationsformen umfassend diskutieren. In diesem Kontext sollten vor allem auch die Einstellungen, Verhaltenserwartungen und Kompetenzen verschiedener schulischer Akteursgruppen hinsichtlich der Kommunikation und Kooperation mit digitalen Medien, die konkrete Ausgestaltung in der Schulpraxis sowie die Einbettung in die schulischen Strukturen und Prozesse in den Blick genommen werden. Für die drei in diesem Beitrag fokussierten Ebenen (Eltern, Lehrpersonen, Schule) lassen sich zusammenfassend die folgenden Forschungsbedarfe formulieren: 
Ebene der Eltern

Die Überzeugungen von Eltern zur digitalen Kommunikation und Zusammenarbeit sind tiefer gehend in den Blick zu nehmen - hier liegen im deutschsprachigen Raum nur wenige Ergebnisse vor. Decken sich die berichteten Potentiale, Bedenken und Ängste mit vorliegenden Ergebnissen aus anderen Ländern?

Die Dokumentation von schulischen Nutzungsszenarien und elterlichen Erfahrungen mit der digitalen Kommunikation und Kooperation ist von Bedeutung. Es ist zu untersuchen, welche konkreten Veränderungen sich ergeben, bezogen darauf wer (mit welchem sozio-ökonomischen Hintergrund, Geschlecht bspw.), wie (in welcher Qualität der Anfragen, auf welchen Kommunikationswegen), worüber (mit welchen Interessen und Anliegen) und in welcher Form (Frequenz, zeitliche Rhythmen) kommuniziert. Verändert sich bei Nutzung digitaler Medien die Qualität und/oder Quantität der Kommunikation und Zusammenarbeit? Im Hinblick auf eine sprachlich, kulturell und sozio-ökonomisch diverse Elternschaft ist zu klären, wie diese durch eine digitale Kommunikation besser erreicht werden kann. Welche Effekte hat beispielsweise die digitale Kommunikation bei Vorhandensein von Sprachbarrieren?

Daneben könnte die Untersuchung geschlechtsspezifischer Aspekte interessant sein, beispielsweise die Effekte der zunehmenden Nutzung digitaler Medien für die Zusammenarbeit zwischen Elternhaus und Schule auf die Quantität und Qualität der Beteiligung von Müttern und Vätern an den Bildungsprozessen ihrer Kinder.

Die Medienkompetenz von Eltern bezogen auf die aktive Gestaltung und Partizipation an digital unterstützten Kommunikations- und Kooperationsprozessen bedarf einer besonderen Aufmerksamkeit der Forschung, da diese Frage stark mit Aspekten von Chancengerechtigkeit zusammenhängt. Dies wird umso dringlicher, wenn auch Lehr-Lernprozesse zunehmend in digitalen Räumen stattfinden. Welche neuen Anforderungen werden an die Medienkompetenz von Eltern gestellt und wie werden diese bewältigt?

\section{Ebene der Lehrpersonen}

Es fehlen Studien zur konkreten Nutzung digitaler Medien in der Kommunikation zwischen Lehrkräften und Eltern. Welche Kommunikationskanäle wählen Lehrkräfte für welche Inhalte? Welche Rolle spielen Einflussfaktoren wie Alter und Geschlecht der Lehrkraft für die Nutzung digitaler Medien als Kommunikationsmittel?

Darüber hinaus sind die Konsequenzen oder Wirkungen einer digitalen Kommunikation zu untersuchen. Inwiefern verändern sich Kommunikationsprozesse durch die Nutzung digitaler Medien? Wird mehr oder weniger Feedback gegeben? Entfallen bestimmte Inhalte oder werden andere Inhalte stärker betont? Werden digitale Medien zur Rückkopplung mit Eltern genutzt, etwa über elektronische Befragungen der Elternschaft, digitale Abstimmungstools oder virtuelle «Kummerkästen»? 
Bezogen auf das Belastungsempfinden der Lehrkräfte stellt sich etwa die Frage, unter welchen Bedingungen die digitale Kommunikation in der Wahrnehmung der Lehrkräfte zu einer Be- oder Entlastung führt. Hier sollten auch zusätzliche Belastungen, die sich im Zuge der COVID-19 Pandemie ergeben haben, in den Blick genommen werden, wie etwa die parallele Kommunikation mit Elternhaus und Lernenden in Zeiten des Wechselunterrichts.

Die Überzeugungen von Lehrkräften zur digital vermittelten Kommunikation und Kooperation mit Eltern sind tiefer gehend zu untersuchen, da sie eine bedeutsame Nutzungsbarriere darstellen. Welche Vor- und Nachteile werden formuliert? Welche Ängste sind hinsichtlich einer digital unterstützten Kommunikation und Kooperation vorhanden? Studien aus unterschiedlichen Ländern zeigen, dass typische Ängste von Lehrkräften sich z. B. darauf richten, dass sie sichtbare «digitale Spuren» hinterlassen oder gewohnte Kommunikationsstrukturen vernachlässigt werden. Hier fehlen Daten für den deutschsprachigen Raum. Auch die Erfassung und Dokumentation von Best-Practice-Beispielen wäre sinnvoll, um Handlungsmöglichkeiten offen zu legen.

Darüber hinaus ist zu untersuchen, inwiefern die Medienkompetenz der Lehrkräfte die (digitale) Kommunikation mit den Eltern bedingt. Welche neuen Kompetenzen benötigen Lehrkräfte und sind diese in die Lehrerausbildung integriert? Welche Rolle spielt etwa die Vermittlung von Kompetenzen zur (digitalen) Gesprächsführung und zum Umgang mit digitalen Medien an den Hochschulen und im Rahmen von Fortbildungen?

\section{Ebene der Schule}

Auf der Ebene institutioneller Strukturen bedarf es im deutschsprachigen Raum umfassenderer Analysen zu den Informations- und Kommunikationsbedürfnissen von Schule und Eltern sowie zur allgemeinen Verfügbarkeit mobiler digitaler Geräte und Anwendungen (z. B. Apps, Online-Systeme) für alle an der digitalen Kommunikation Beteiligten. Technische Aspekte (z. B. die Wartung von Plattformen und Geräten, der Versand von elektronischen Lehrerbriefen etc.) sowie generell die Abhängigkeit von einer entsprechenden ICT-Infrastruktur (z. B. Anschluss an Glasfasernetze, um Videotelefonie zu ermöglichen) können einen erheblichen Einfluss auf die Kommunikationsflüsse nehmen - und auf deren Wahrnehmung als reibungslose Prozesse. Die technischen Voraussetzungen betreffen dabei nicht nur die Schule, sondern auch die häusliche Ausstattung der Lehrkräfte und Eltern.

Zudem sollte die Forschung Fragen des Datenschutzes und der Umsetzung rechtlicher Bedingungen im Kontext von Kommunikation und Kooperation zwischen Schule und Elternhaus stärker in den Blick nehmen (Ifenthaler u. a. 2020). Welche Inhalte dürfen über welche Plattformen weitergegeben werden? Wie ist der Zugriff auf Kommunikationsressourcen für verschiedene Akteursgruppen geregelt? Inwiefern nehmen Zugriffsrechte einen Einfluss darauf, wer welche Kommunikationsform überhaupt und wie häufig benutzt? 
Auch sollten weitere Schulakteure in den Blick genommen werden, wie beispielsweise die Perspektiven von Schülerinnen und Schüler oder die Rolle von IT-Administratorinnen und -Administratoren.

Auf schulkultureller Ebene ist zu untersuchen, in welchem Ausmass eine existierende Kommunikations- und Kooperationskultur die digitale Zusammenarbeit prägt und wie sich diese umgekehrt auch durch die Besonderheiten digitaler Kommunikation verändern kann (etwa im Kontext der Ganztagsschule). Wie kann die Einführung neuer digital unterstützter Kommunikationsformen in den Schulen optimal begleitet und kritisch reflektiert werden?

Methodisch bedarf es sowohl quantitativer als auch qualitativer Zugänge, um die Kommunikations- und Kollaborationsprozesse mit digitalen Medien sichtbar zu machen und die verschiedenen Konsequenzen abzuschätzen. Mündliche und schriftliche Befragungen von Lehrpersonen, Eltern und weiteren Akteurinnen und Akteuren bieten beispielsweise Hinweise auf Überzeugen, Ängste, Kompetenzen und Nutzungserfahrungen im Hinblick auf die digitale Kommunikation. Neben querschnittlichen wären hier vor allem auch längsschnittliche Daten wichtig, um die Veränderungen in der Kommunikationskultur bei Einführung einer digitalen Kommunikationsplattform zu dokumentieren und die Ursache-Wirkungs-Zusammenhänge abschätzen zu können. Darüber hinaus sollten neue forschungsmethodische Möglichkeiten der Messung der Qualität der Kommunikation und Kooperation genutzt werden, die über die persönliche Wahrnehmung der Befragten hinausgehen. Von Interesse wären beispielsweise Materialanalysen von Kommunikationsprotokollen - insbesondere im Vergleich unterschiedlicher digital unterstützter Kommunikationsmedien im Hinblick auf Inhalte und Anliegen, Sprachstil usw. In diesem Kontext sind forschungsmethodischethische Aspekte zu klären, die z. B. Analysen von Kommunikationsprotokollen mit sich bringen (etwa, wenn diese Schülerdaten enthalten). Von Interesse könnte auch die Untersuchung eines Multi-Step-Flow of Communication sein - bspw. der digitale Informationsfluss von der Schulleitung oder den Lehrkräften über die (Klassen-) Elternsprecherinnen und -Elternsprecher hin zu den Eltern. Die Rolle dieser Mittelsleute in der Kommunikation und auch die genutzten, möglicherweise diversen Kommunikationsinstrumente könnten genauer in den Blick genommen werden.

In Bezug auf die eingangs herausgearbeiteten Optimierungsunterstellungen in Strategiepapieren stellt sich die Frage, in welcher Form Empfehlungen zur digitalen Kommunikation und Zusammenarbeit zwischen Eltern und Schule gegeben werden können - insbesondere, wenn man sich die Bedeutung der Zusammenarbeit mit Eltern vor Augen hält. 


\section{Literatur}

Aich, Gernot, Christina Kuboth, Martin Gartmeier, und Daniela Sauer, Hrsg. 2017. Kommunikation und Kooperation mit Eltern. Weinheim/Basel: Beltz.

Alanko, Anu. 2018. «Preparing Pre-Service Teachers for Home-School Cooperation: Exploring Finnish Teacher Education Programmes». Journal of Education for Teaching 44 (3): 321-32. https://doi.org/10.1080/02607476.2018.1465644.

Autorengruppe Paducation. 2014. Paducation. Evaluation eines Modellversuchs mit Tablets am Hamburger Kurt-Körber Gymnasium. Bremen/Hamburg: Institut für Informationsmanagement Bremen GmbH, Universität Hamburg.

Bastian, Jasmin. 2017. «Tablets zur Neubestimmung des Lernens?». In Tablets in Schule und Unterricht: Forschungsmethoden und -perspektiven zum Einsatz digitaler Medien, herausgegeben von Jasmin Bastian und Stefan Aufenanger, 139-73. Wiesbaden: Springer. https:// doi.org/10.1007/978-3-658-13809-7_7.

Bastian, Jasmin, und Stefan Aufenanger, Hrsg. 2017. Tablets in Schule und Unterricht: Forschungsmethoden und -perspektiven zum Einsatz digitaler Medien. VS Verlag für Sozialwissenschaften. https://doi.org/10.1007/978-3-658-13809-7.

Blau, Ina, und Mira Hameiri. 2012. «Teacher-Families Online Interactions and Gender Differences in Parental Involvement Through School Data System: Do Mothers Want to Know More than Fathers about Their Children?». Computers \& Education 59 (2): 701-9. https:// doi.org/10.1016/j.compedu.2012.03.012.

Blau, Ina, und Mira Hameiri. 2017. «Ubiquitous Mobile Educational Data Management by Teachers, Students and Parents: Does Technology Change School-Family Communication and Parental Involvement?». Education and Information Technologies 22 (3): 1231-47. https://doi.org/10.1007/s10639-016-9487-8.

Bol, Thijs. 2020. «Inequality in Homeschooling during the Corona Crisis in the Netherlands. First Results from the LISS Panel». https://doi.org/10.31235/osf.io/hf32q.

Boonk, Lisa, Hieronymus J. M. Gijselaers, Henk Ritzen, und Saskia Brand-Gruwel. 2018. «A Review of the Relationship between Parental Involvement Indicators and Academic Achievement». Educational Research Review 24 (Juni): 10-30. https://doi.org/10.1016/j.edurev.2018.02.001.

Börner, Nicole, Stefan Eberitzsch, Ramona Grotheus, und Agathe Wilk. 2011. «Bildungsbericht Ganztagsschule NRW 2011». Dortmund: Eigenverlag Forschungsverbund DJI/TU Dortmund.

Bouffard, Suzanne M. 2006. «Virtual Parental Involvement: The Role of the Internet in ParentSchool Communications». Duke University. https://docplayer.net/14834568-Virtual-parental-involvement-the-role-of-the-internet-in-parent-school-communications.html.

Bouffard, Suzanne M. 2008. "Tapping Into Technology: The Role of the Internet in FamilySchool Communication». Harvard Family Research Project. https://archive.globalfrp.org/ publications-resources/browse-our-publications/tapping-into-technology-the-role-ofthe-internet-in-family-school-communication. 
Breiter, Andreas, Angelina Lange, und Emese Stauke. 2008. School Information System and Data-Based Decision-Making. Schulinformationssysteme und datengestützte Entscheidungsprozesse. Bilingual Edition. Frankfurt am Main/New York: Peter Lang.

Breiter, Andreas, und Arne Hendrik Ruhe. 2018. «Paper Versus School Information Management Systems: Governing the Figurations of Mediatized Schools in England and Germany». In Communicative Figurations: Transforming Communications in Times of Deep Mediatization, herausgegeben von Andreas Hepp, Andreas Breiter, und Uwe Hasebrink, 313-39. Cham: Palgrave Macmillan. https://doi.org/10.1007/978-3-319-65584-0_13.

Bremer, Claudia, und Alexander Tillmann. 2017. «Einsatz von Tablets in Grundschulen. Umsetzung und Ergebnisse des Projektes Mobiles Lernen in Hessen (MOLE)». In Tablets in Schule und Unterricht, herausgegeben von Jasmin Bastian und Stefan Aufenanger, 241-76. Wiesbaden: Springer Fachmedien. https://doi.org/10.1007/978-3-658-13809-7_11.

Castro, Dina C., Donna M. Bryant, Ellen S. Peisner-Feinberg, und Martie L. Skinner. 2004. «Parent Involvement in Head Start Programs: The Role of Parent, Teacher and Classroom Characteristics». Early Childhood Research Quarterly 19 (3): 413-30. https://doi.org/10.1016/j. ecresq.2004.07.005.

Clinton, Janet, John Hattie, und Robyn Dixon. 2007. Evaluation of the Flaxmere Project: When Families Learn the Language of School. Wellington, N.Z.: Ministry of Education, New Zealand.

Daft, Richard L., und Robert H. Lengel. 1986. "Organizational Information Requirements, Media Richness and Structural Design». Management Science 32 (5): 554-71. https://doi. org/10.1287/mnsc.32.5.554.

Desforges, Charles, und Alberto Abouchaar. 2003. «The Impact of Parental Involvement, Parental Support and Family Education on Pupil Achievement and Adjustment: A Literature Review». Herausgegeben von Department for Education and Skills.

Döbeli Honegger, Beat. 2017. Mehr als 0 und 1: Schule in einer digitalisierten Welt. 2. Auflage. Bern: Hep. http://www.mehrals0und1.ch.

Dor, Asnat. 2018. «Parent-Teacher Communication: The Case of Diverse Family Patterns». Education and Society 36 (1): 5-20. https://doi.org/10.7459/es/36.1.02.

Drossel, Kerstin, Birgit Eickelmann, Heike Schaumburg, Amelie Labusch, Heike Schaumburg, Knut Schwippert, Martin Senkbeil, Jan Vahrenhold. 2019. «Nutzung digitaler Medien und Prädiktoren aus der Perspektive der Lehrerinnen und Lehrer im internationalen Vergleich». In ICILS 2018 \#Deutschland. Computer- und informationsbezogene Kompetenzen von Schülerinnen und Schülern im zweiten internationalen Vergleich und Kompetenzen im Bereich Computational Thinking, 205-40. Münster: Waxmann. https://doi.org/10.25656/01:18325.

Dumont, Hanna, Ulrich Trautwein, Gabriel Nagy, und Benjamin Nagengast. 2014. "Quality of Parental Homework Involvement: Predictors and Reciprocal Relations with Academic Functioning in the Reading Domain». Journal of Educational Psychology 106 (1): 144-61. https://doi.org/10.1037/a0034100. 
Eickelmann, Birgit, Wilfried Bos, Julia Gerick, Frank Goldhammer, Heike Schaumburg, Knut Schwippert, Martin Senkbeil, Jan Vahrenhold, Hrsg. 2019. ICILS 2018 \#Deutschland computer- und informationsbezogene Kompetenzen von Schülerinnen und Schülern im zweiten internationalen Vergleich und Kompetenzen im Bereich Computational Thinking. Münster: Waxmann. https://doi.org/10.25656/01:18166.

Eickelmann, Birgit, und Kerstin Drossel. 2020. «Schule auf Distanz. Perspektiven und Empfehlungen für den neuen Schulalltag: Eine repräsentative Befragung von Lehrkräften in Deutschland». Düsseldorf: Vodafone Stiftung Deutschland.

Epstein, Joyce L. 1995. «School/Family/Community Partnerships: Caring for the Children We Share». Phi Delta Kappan 76 (9): 701-12.

Epstein, Joyce L., Mavis G. Sanders, Steven B. Sheldon, Beth S. Simon, Karen Clark Salinas, Natalie Rodriguez Jansorn, Frances L. Van Voorhis, u. a. 2018. School, Family, and Community Partnerships: Your Handbook for Action. Fourth Edition. Corwin.

Ertmer, Peggy, Anne Ottenbreit-Leftwich, und Jo Tondeur. 2015. «Teacher Beliefs and Uses of Technology to Support 21st Century Teaching and Learning». In International Handbook of Research on Teachers' Beliefs, 403-19. Routledge. http://hdl.handle.net/1854/LU-5815883.

Fan, Weihua, und Cathy M. Williams. 2010. «The Effects of Parental Involvement on students' Academic Self-Efficacy, Engagement and Intrinsic Motivation». Educational Psychology 30 (1): 53-74. https://doi.org/10.1080/01443410903353302.

Gomolla, Mechtild, und Carolin Rotter. 2014. "Zugewanderte und einheimische Eltern: Gemeinsamkeiten und Unterschiede in der Beurteilung von Schulpolitik und -praxis». In Eltern ziehen Bilanz. Ein Trendbericht zu Schule und Bildungspolitik in Deutschland, herausgegeben von Dagmar Killus und Klaus-Jürgen Tillmann. Waxmann Verlag.

Green, Christa L., Joan M. T. Walker, Kathleen V. Hoover-Dempsey, und Howard M. Sandler. 2007. «Parents' Motivations for Involvement in Children's Education: An Empirical Test of a Theoretical Model of Parental Involvement». Journal of Educational Psychology 99 (3): 532-44. https://doi.org/10.1037/0022-0663.99.3.532.

Hall, Elaine, Kate Wall, Steve Higgins, Linda Stephens, Irene Pooley, und John Welham. 2005. «Learning to Learn with Parents: Lessons from Two Research Projects». Improving Schools 8 (2): 179-91. https://doi.org/10.1177/1365480205057706.

Hattie, John. 2009. Visible Learning: a Synthesis of over 800 Meta-Analyses Relating to Achievement. London/New York: Routledge.

Hawighorst, Britta. 2009. «Perspektiven von Einwandererfamilien». In Migration und schulischer Wandel: Elternbeteiligung, herausgegeben von Sara Fürstenau und Mechtild Gomolla, 51-67. Wiesbaden: VS Verlag für Sozialwissenschaften. https://doi.org/10.1007/978-3-53191487-9_3.

Heath, Don, Rozan Maghrabi, und Nora Carr. 2015. «Implications of Information and Communication Technologies (ICT) for School-Home Communication». Journal of Information Technology Education: Research 14: 363-95. https://doi.org/10.28945/2285. 
Hertel, Silke, Simone Bruder, und Bernhard Schmitz. 2009. «Beratungs- und Gesprächsführung von Lehrkräften». In Lehrprofessionalität: Bedingungen, Genese, Wirkungen und ihre Messung, herausgegeben von Olga Zlatkin-Troitschanskaia und Klaus Beck, Detlef Sembill, Reinhold Nickolaus, und Regina Mulder, 117-28. Weinheim: Beltz.

Hertel, Silke. 2009. Beratungskompetenz von Lehrern: Kompetenzdiagnostik, Kompetenzförderung, Kompetenzmodellierung. Pädagogische Psychologie und Entwicklungspsychologie 74. Münster: Waxmann.

Hertel, Silke, Nina Jude, und Johannes Naumann. 2010. «Leseförderung im Elternhaus». In PISA 2009. Bilanz nach einem Jahrzehnt, herausgegeben von Eckhard Klieme, Cordula Artelt, Wolfgang Schneider, Petra Stanat, Johannes Hartig, Nina Jude, Olaf Köller, und Manfred Prenzel, 255-75. Münster: Waxmann Verlag. https://doi.org/10.25656/01:3538.

Hertel, Silke, Nina Jude, und Christine Sälzer. 2017. «Empirische Arbeit: Perspektiven von Eltern auf die Zusammenarbeit mit der Schule: Differenzierte Analysen für den Sekundarschulbereich auf Basis von Daten aus PISA 2012». Psychologie in Erziehung und Unterricht 64 (4): 243-55. https://doi.org/10.2378/peu2017.art21d.

Hertzsch, Helen und Elke Dörflinger. 2018. «Beratung im und für das Schulsystem». In Kommunikation in der Schule, herausgegeben von Ines C. Vogel, 2., aktualisierte Auflage, 297-322. Bad Heilbrunn: Verlag Julius Klinkhardt.

Hertzsch, Helen und F. M. Schneider. 2018. «Kommunikationskompetenz von Lehrkräften an Schulen». In Kommunikation in der Schule, herausgegeben von Ines C. Vogel, 2., aktualisierte Auflage, 75-100. Bad Heilbrunn: Verlag Julius Klinkhardt.

Hilkenmeier, Johanna, Sabrina Wiescholek, und Heike M. Buhl. 2017. «Empirische Arbeit: Motivationsförderung am Elternsprechtag. Zum Zusammenhang zwischen eingeschätzter Leseleistung, Lehrer- und Elternverhalten». Psychologie in Erziehung und Unterricht 64 (4): 256-69. https://doi.org/10.2378/peu2017.art18d.

Hill, Nancy E., und Diana F. Tyson. 2009. «Parental Involvement in Middle School: A Meta-Analytic Assessment of the Strategies That Promote Achievement». Developmental Psychology 45 (3): 740-63. https://doi.org/10.1037/a0015362.

Hornby, Garry, und Rayleen Lafaele. 2011. «Barriers to Parental Involvement in Education: an Explanatory Model». Educational Review 63 (1): 37-52. https://doi.org/10.1080/00131911. 2010.488049.

Huber, Stephan Gerhard, Paula Sophie Günther, Nadine Schneider, Christoph Helm, Marius Schwander, Jane Pruitt, und Julia Schneider. 2020. COVID-19 und aktuelle Herausforderungen in Schule und Bildung. Erste Befunde des Schul-Barometers in Deutschland, Österreich und der Schweiz. Waxmann Verlag. https://doi.org/10.25656/01:20579.

Ifenthaler, Dirk, David Gibson, Doreen Prasse, Atsushi Shimada, und Masanori Yamada. 2020. «Putting Learning Back into Learning Analytics: Actions for Policy Makers, Researchers, and Practitioners». Educational Technology Research and Development, 1-20. https://doi. org/10.1007/s11423-020-09909-8.

Jeynes, William. 2005. «Effects of Parental Involvement and Family Structure on the Academic Achievement of Adolescents». Marriage and Family Review 37 (Juli): 99-116. https://doi. org/10.1300/J002v37n03_06. 
KMK. 2016. «Bildung in der digitalen Welt. Strategie der Kultusministerkonferenz». Berlin. https://www.kmk.org/themen/bildung-in-der-digitalen-welt/strategie-bildung-in-der-digitalen-welt.html.

KMK. 2018a. «Bildung und Erziehung als gemeinsame Aufgabe von Eltern und Schule. Beschluss der Kultusministerkonferenz vom 11.10.2018». https://www.kmk.org/fileadmin/ Dateien/veroeffentlichungen_beschluesse/2018/2018_10_11-Empfehlung-Bildung-undErziehung.pdf.

KMK. 2018b. «Bildung und Erziehung als gemeinsame Aufgabe von Eltern und Schule. Informationen der Länder über die Zusammenarbeit von Eltern und Schule. Beschluss der KMK vom 04.12.2003 i. d. F. vom 11.10.2018». https://www.kmk.org/fileadmin/Dateien/veroeffentlichungen_beschluesse/2018/2018_10_11-Dokumentation-Bildung-und-Erziehung. pdf.

Kraft, Matthew A., und Shaun M. Dougherty. 2013. «The Effect of Teacher-Family Communication on Student Engagement: Evidence From a Randomized Field Experiment». Journal of Research on Educational Effectiveness 6 (3): 199-222. https://doi.org/10.1080/19345747.20 12.743636.

Kuusimäki, Anne-Mari, Lotta Uusitalo-Malmivaara, und Kirsi Tirri. 2019. «Parents' and Teachers' Views on Digital Communication in Finland». Education Research International 2019 (Juli): 1-7. https://doi.org/10.1155/2019/8236786.

Lee, Jung-Sook, und Natasha K. Bowen. 2006. «Parent Involvement, Cultural Capital, and the Achievement Gap Among Elementary School Children». American Educational Research Journal 43 (2): 193-218. https://doi.org/10.3102/00028312043002193.

Lee, Paul, Louis Leung, Ven-Hwei Lo, Chengyu Xiong, und Tingjun Wu. 2011. «Internet Communication Versus Face-to-face Interaction in Quality of Life». Social Indicators Research 100 (Februar): 375-89. https://doi.org/10.1007/s11205-010-9618-3.

Lewin, Cathy, und Rosemary Luckin. 2010. «Technology to Support Parental Engagement in Elementary Education: Lessons Learned from the UK». Computers \& Education 54 (3): 749 58. https://doi.org/10.1016/j.compedu.2009.08.010.

Lorenz, F., und Elke Wild. 2007. «Parental Involvement in Schooling - Results Concerning Its Structure and Impact on Students' Motivation». In Studies on the Educational Quality of Schools. The Final Report on the DFG Priority Programme. https://pub.uni-bielefeld.de/record/2904657.

Macia Bordalba, Monica, und Jordi Garreta Bochaca. 2019. «Digital Media for Family-School Communication? Parents' and Teachers' Beliefs». Computers \& Education 132 (April): 4462. https://doi.org/10.1016/j.compedu.2019.01.006.

Manolev, Jamie, Anna Sullivan, und Roger Slee. 2019. «The Datafication of Discipline: ClassDojo, Surveillance and a Performative Classroom Culture». Learning, Media and Technology 44 (1): 36-51. https://doi.org/10.1080/17439884.2018.1558237.

Murray, Elizabeth, Laura McFarland-Piazza, und Linda J. Harrison. 2015. «Changing Patterns of Parent-Teacher Communication and Parent Involvement from Preschool to School». Early Child Development and Care 185 (7): 1031-52. https://doi.org/10.1080/03004430.2014.975 223. 
Natale, Kirstin, und Kathryn Lubniewski. 2018. «Use of Communication and Technology among Educational Professionals and Families». International Electronic Journal of Elementary Education 10 (3): 377-84. https://doi.org/10.26822/iejee.2018336196.

Neuenschwander, Markus, Thomas Balmer, Annette Gasser, Stefanie Goltz, Ueli Hirt, Hans Ryser, und Hermann Wartenweiler. 2004. «Eltern, Lehrpersonen und Schülerleistungen. Schlussbericht». Bern: Kanton und Universität Bern. http://www.fhnw.ch/ppt/content/ pub/fase-b-welle-1-eltern-lehrpersonen-und-schuelerleistungen/artikel-pdf.

Olmstead, Christine. 2013. «Using Technology to Increase Parent Involvement in Schools». TechTrends 57 (6): 28-37. https://doi.org/10.1007/s11528-013-0699-0.

Otterpohl, Nantje, und Elke Wild. 2017. «Kooperation zwischen Elternhaus und Schule im Kontext der schulischen Leistungsentwicklung». In Handbuch Entwicklungs- und Erziehungspsychologie, herausgegeben von Bärbel Kracke und Peter Noack, 1-15. Springer Reference Psychologie. Berlin/Heidelberg: Springer. https://doi.org/10.1007/978-3-642-54061-5_131.

Palts, Karmen, und Veronika Kalmus. 2015. «Digital Channels in Teacher-Parent Communication: The Case of Estonia». The International Journal of Education and Development Using Information and Communication Technology 11 (Dezember): 65-81.

Park, Sira, und Susan D. Holloway. 2017. «The Effects of School-Based Parental Involvement on Academic Achievement at the Child and Elementary School Level: A Longitudinal Study». The Journal of Educational Research 110 (1): 1-16. https://doi.org/10.1080/00220671.2015 .1016600 .

Pekrun, Reinhard. 2001. «Familie, Schule und Entwicklung». In Familie und Entwicklung: aktuelle Perspektiven der Familienpsychologie, herausgegeben von Sabine Walper und Reinhard Pekrun, 84-105. Göttingen: Hogrefe.

Penney, Sharon, Gabrielle Young, Kimberly Maich, und Emily Butler. 2019. «Förderung der Kommunikation zwischen Elternhaus und Schule um ein Kind mit Beeinträchtigung: Wahrnehmungen von Eltern in Neufundland und Labrador». In Inklusion in Kanada. Internationale Perspektiven auf heterogenitätssensible Bildung, herausgegeben von David Jahr und Robert Kruschel, 207-23. Weinheim: Beltz.

Perelman, Uri. 2014. «What are the Relationships between Teachers' Engagement with Management Information Systems and their Sense of Accountability?» Interdisciplinary Journal of E-Learning and Learning Objects 10: 217-27.

Petko, Dominik. 2012. «Teachers' Pedagogical Beliefs and their Use of Digital Media in Classrooms: Sharpening the Focus of the 'Will, Skill, Tool' Model and Integrating Teachers' Constructivist Orientations». Computers \& Education 58 (4): 1351-59. https://doi.org/10.1016/j. compedu.2011.12.013.

Prasse, Doreen. 2012. «Bedingungen innovativen Handelns in Schulen. Funktion und Interaktion von Innovationsbereitschaft, Innovationsklima und Akteursnetzwerken am Beispiel der IKT-Integration an Schulen». Empirische Erziehungswissenschaft. 38. Münster: Waxmann. 
Prasse, Doreen, Beat Döbeli Honegger, und Michael Hielscher. 2021. «1:1 Ausstattungen und BYOD-Klassen - Potenziale, Wirkungen und Empfehlungen». In Handbuch Lernen mit digitalen Medien. Weinheim: Beltz.

Prasse, Doreen, Beat Döbeli Honegger, und Dominik Petko. 2017. «Digitale Heterogenität von Lehrpersonen: Herausforderung oder Chance für die ICT-Integration in Schulen?» Beiträge zur Lehrerinnen- und Lehrerbildung 35: 219-33. https://doi.org/10.5167/UZH-170423.

Prasse, Doreen, Nives Egger, Andrea Cantieni, Martin Hermida, und Nina Iten. 2020. Lernen und Unterrichten in Tabletklassen 2016 bis 2019. Abschlussbericht zur wissenschaftlichen Begleitforschung. Goldau: Institut für Medien und Schule, PH Schwyz. www.phsz.ch/forschung/medien-und-schule/lernen-mit-tablets/.

Prasse, Doreen, Nives Egger, und Beat Döbeli Honegger. 2017. «Mobiles Lernen. Auch zu Hause?» In Tablets in Schule und Unterricht, herausgegeben von Jasmin Bastian und Stefan Aufenanger, 209-39. Wiesbaden: Springer. https://doi.org/10.1007/978-3-658-13809-7_10.

Sacher, Werner. 2004. Elternarbeit in den bayerischen Schulen: Repräsentativ-Befragung zur Elternarbeit im Sommer 2004; erster Übersichtsbericht. Schulpädagogische Untersuchungen Nürnberg 23. Nürnberg: Universität.

Sacher, Werner. 2005. Erfolgreiche und misslingende Elternarbeit: Ursachen und Handlungsmöglichkeiten; erarbeitet auf der Grundlage der Repräsentativbefragung an bayerischen Schulen im Sommer 2004. Schulpädagogische Untersuchungen Nürnberg 24. Nürnberg: Universität.

Sacher, Werner. 2006. «Elternhaus und Schule. Bedingungsfaktoren ihres Verhältnisses, aufgezeigt an der bayerischen Studie vom Sommer 2004». Bildung und Erziehung 59 (3), 303-22.

Sacher, Werner. 2008. Elternarbeit: Gestaltungsmöglichkeiten und Grundlagen für alle Schularten. Bad Heilbrunn: Klinkhardt.

Sacher, Werner. 2014. Elternarbeit als Erziehungs- und Bildungspartnerschaft: Grundlagen und Gestaltungsvorschläge für alle Schularten. 2., vollständig überarbeitete Auflage. Bad Heilbrunn: Klinkhardt.

Schaumburg, Heike. 2018. «Empirische Befunde zur Wirksamkeit unterschiedlicher Konzepte des digital unterstützten Lernens». In Digitalisierung in der schulischen Bildung. Chancen und Herausforderungen, herausgegeben von Nele McElvany, Franziska Schwabe, Wilfried Bos, und Heinz G. Holtappels, 27-40. Münster: Waxmann.

Schaumburg, Heike, und Doreen Prasse. 2019. Medien und Schule: Theorie - Forschung - Praxis. Bad Heilbrunn: Klinkhardt.

Schu, Cornelia, Hrsg. 2014. «Eltern als Bildungspartner. Wie Beteiligung an Grundschulen gelingen kann». Berlin: Forschungsbereich beim Sachverständigenrat deutscher Stiftungen für Integration und Migration.

Selwyn, Neil, Shakuntala Banaji, Christina Hadjithoma-Garstka, und Wilma Clark. 2011. «Providing a Platform for Parents? Exploring the Nature of Parental Engagement with School Learning Platforms: Providing a Platform for Parents?» Journal of Computer Assisted Learning 27 (4): 314-23. https://doi.org/10.1111/j.1365-2729.2011.00428.x. 
Smith, Tyler E., Susan M. Sheridan, Elizabeth M. Kim, Sunyoung Park, und S. Natasha Beretvas. 2020. "The Effects of Family-School Partnership Interventions on Academic and SocialEmotional Functioning: A Meta-Analysis Exploring What Works for Whom». Educational Psychology Review 32 (2): 511-44. https://doi.org/10.1007/s10648-019-09509-w.

Stange, Waldemar, Rolf Krüger, Angelika Henschel, und Christof Schmitt, Hrsg. 2012. Erziehungs- und Bildungspartnerschaften: Grundlagen und Strukturen von Elternarbeit. Wiesbaden: VS Verlag für Sozialwissenschaften. https://doi.org/10.1007/978-3-531-94279-7.

Tengler, Karin, Natalie Schrammel, und Gerhard Brandhofer. 2020. «Lernen trotz Corona. Chancen und Herausforderungen des distance learning an österreichischen Schulen». Medienimpulse 58 (2). https://doi.org/10.21243/mi-02-20-24.

Thompson, Blair. 2008. «Characteristics of Parent-Teacher E-Mail Communication». Communication Education 57 (2): 201-23. https://doi.org/10.1080/03634520701852050.

Thompson, Mazer, und Elizabeth Flood-Grady. 2015. «The Changing Nature of Parent-Teacher Communication: Mode Selection in the Smartphone Era». Communication Education 64 (April): 187-207. https://doi.org/10.1080/03634523.2015.1014382.

Tulodziecki, Gerhard, Silke Grafe, und Bardo Herzig. 2019. Medienbildung in Schule und Unterricht: Grundlagen und Beispiele. 2. Aufl. Bad Heilbrunn: Klinkhardt.

Venkatesh, Viswanath, Michael Morris, Gordon Davis, und Fred Davis. 2003. «User Acceptance of Information Technology: Toward a Unified View». MIS Quarterly 27 (September): 425-78. https://doi.org/10.2307/30036540.

Venkatesh, Viswanath, James Y. L. Thong, und Xin Xu. 2012. «Consumer Acceptance and Use of Information Technology: Extending the Unified Theory of Acceptance and Use of Technology». MIS Quarterly 36 (1): 157-78. https://doi.org/10.2307/41410412.

Walper, Müller, und Kleinschrot. 2019. «Erziehungs- und Bildungspartnerschaft in der Schule als Herausforderung für Eltern und Lehrkräfte». In Qualität, Professionalisierung und Monitoring im Bildungssystem, herausgegeben von Hartmut Ditton und Rudolf Tippelt, 147-63. Münster: Waxmann.

Wasserman, Egoza, und Yaffa Zwebner. 2017. «Communication between Teachers and Parents Using the WhatsApp Application». International Journal of Learning, Teaching and Educational Research 16 (12): 1-12. https://doi.org/10.26803/ijlter.16.12.1.

Welling, Stefan, Andreas Breiter, und Arne Hendrik Schulz. 2014. Mediatisierte Organisationswelten in Schulen: Wie der Medienwandel die Kommunikation in den Schulen verändert. Wiesbaden: Springer VS. https://doi.org/10.1007/978-3-658-03677-5.

Wild, Elke, und Fiona Lorenz. 2010. Elternhaus und Schule. https://nbn-resolving.org/ urn:nbn:de:101:1-201308056359.

Yotyodying, Sittipan, und Elke Wild. 2014. "Antecedents of Different Qualities of HomeBased Parental Involvement: Findings from a Cross-Cultural Study in Germany and Thailand». Learning, Culture and Social Interaction 3 (2): 98-110. https://doi.org/10.1016/j. Icsi.2014.02.002. 